\title{
Tunable Doniach phase diagram for strongly-correlated nanoclusters
}

\author{
Yan Luo,* C. Verdozzi, and Nicholas Kioussis \\ Department of Physics, California State University, Northridge, California 91330-8268, USA
}

(Received 1 May 2004; published 5 January 2005)

\begin{abstract}
Exact diagonalization calculations reveal that the energy spacing $\Delta$ in the conduction band tunes the interplay between the local Kondo and nonlocal Ruderman-Kittel-Kasuya-Yosida (RKKY) interactions, giving rise to a Doniach phase diagram for a nanocluster with regions of prevailing Kondo or RKKY correlations. The parity of the total number of electrons alters the competition between the Kondo and RKKY correlations. This interplay may be relevant to experimental realizations of small rings or quantum dots with tunable magnetic properties. Below a critical value $V_{c}$ of the hybridization the susceptibility exhibits a low- $T$ exponential activation behavior determined by the interplay of the spin gap and $\Delta$.
\end{abstract}

DOI: 10.1103/PhysRevB.71.033304

PACS number(s): 75.20.Hr, 75.75.+a, 75.40.Cx, 75.40.Mg

The interplay between the local Kondo interactions and the nonlocal Ruderman-Kittel-Kasuya-Yosida (RKKY) interactions is a central unresolved problem in the physics of dense valence fluctuation and heavy-fermion compounds. ${ }^{1-3}$ The first interaction, responsible for the quenching of the local $f$ moment via the screening of the conduction electrons, has a characteristic energy scale given by the single-impurity bulk Kondo temperature, $T_{K} \propto \exp \left[-1 / \rho\left(E_{F}\right) J\right]$. ${ }^{4}$ Here, $\rho\left(E_{F}\right)$ is the density of states of the conduction electrons at the Fermi energy and $J$ is the antiferromagnetic interaction between the impurity spin and the conduction electrons. The latter interaction is an indirect magnetic interaction between localized moments mediated by the conduction electrons, with an energy scale of order $T_{R K K Y} \propto J^{2} \rho\left(E_{F}\right)$ which promotes long- or short-range magnetic ordering. ${ }^{1,2,4}$ In the high-temperature regime the localized moments and the conduction electrons retain their identities and interact weakly with each other. At low temperatures, the moments order magnetically if the RKKY interaction is much larger than the Kondo energy, while in the reverse case the system forms a heavy Fermi liquid of quasiparticles that are composites of local moment spins bound to the conduction electrons. ${ }^{3}$ The overall physics can be described by the well-known Doniach phase diagram. ${ }^{1}$ The description of the low-temperature state, when both the RKKY and the Kondo interactions are of comparable magnitude, is an intriguing question that remains poorly understood and is the subject of current active research. ${ }^{3}$

There has been a revival of the Kondo physics in recent years due to an exciting series of experiments on nanoscale systems, which has enabled us to probe local interactions in a well controlled way at the nanoscale. ${ }^{5-8}$ For example, in scanning tunneling microscopy (STM) experiments ${ }^{8}$ the interaction of magnetic impurities with the electrons of a single-wall nanotube confined in one dimension was studied. Interestingly, in addition to the bulk Kondo resonance new subpeaks were found in shortened carbon nanotubes, separated by about the average energy spacing $\Delta$ in the nanotube. These experiments invite an interesting and important question: How does the interplay between the Kondo effect and RKKY interactions become modified in a nanocluster containing magnetic impurities, where the conduction electron spectrum becomes discrete with a mean energy level spacing $\Delta$ ? For such small systems, controlling the magnetism upon tuning the model parameters is acquiring increasing importance, as in ultracold atomic gases on a one-dimensional lattice ${ }^{9}$ and quantum engineering. ${ }^{10}$ While the effect of $\Delta$ on the single-impurity Anderson or Kondo model has received considerable theoretical ${ }^{11-16}$ attention recently, its role on dense impurity clusters remains an unexplored area thus far. The low-temperature behavior of a nanosized heavy-electron system was recently studied within the mean-field approximation. ${ }^{17}$

In this work we present exact diagonalization calculations for $d$ - or $f$-electron nanoclusters to study the effect of energy spacing, parity of the number of electrons, and hybridization on the interplay between the Kondo and RKKY interactions in dense strongly correlated electron nanoclusters. While the properties of the system depend on their geometry and size, ${ }^{18}$ the present calculations treat exactly the Kondo and RKKY interactions. Our results show that (i) tuning $\Delta$ and the parity of the total number of electrons can drive the nanocluster from the Kondo to the RKKY regime, i.e., a zerotemperature energy spacing versus hybridization phase diagram; (ii) the temperature versus hybridization Doniach phase diagram for the nanocluster depends on the energy spacing; and (iii) below a critical value $V_{c}$ of the hybridization the susceptibility exhibits a low- $T$ exponential activation behavior determined by the interplay of the spin gap and $\Delta$. In contrast, above $V_{c}$ there is no exponential behavior.

The one-dimensional Anderson lattice Hamiltonian is

$$
\begin{aligned}
H= & -t \sum_{i \sigma}\left(c_{i \sigma}^{\dagger} c_{i+1 \sigma}+\text { H.c. }\right)+E_{f} \sum_{i \sigma} n_{i \sigma}^{f}+U \sum_{i} n_{i \uparrow}^{f} n_{i \downarrow}^{f} \\
& +V \sum_{i \sigma}\left(f_{i \sigma}^{\dagger} c_{i \sigma}+\text { H.c. }\right)
\end{aligned}
$$

where $t$ is the nearest-neighbor hopping matrix element for the conduction electrons $\left(\epsilon_{k}=-2 t \cos k\right), f_{i, \sigma}\left(c_{i, \sigma}\right)$ annihilates a localized $f$ (conduction) electron on site $i$ with spin $\sigma, E_{f}$ is the energy level of the localized orbital, $V$ is the on-site $f$ - $c$ hybridization matrix element, and $U$ is the on-site Coulomb repulsion of the $f$ electrons. We consider the half-filled $\left(N_{e l}\right.$ $=2 N=12)$ symmetric $\left(E_{f}=-U / 2=-2.5\right)$ case with $N$ sites ar- 


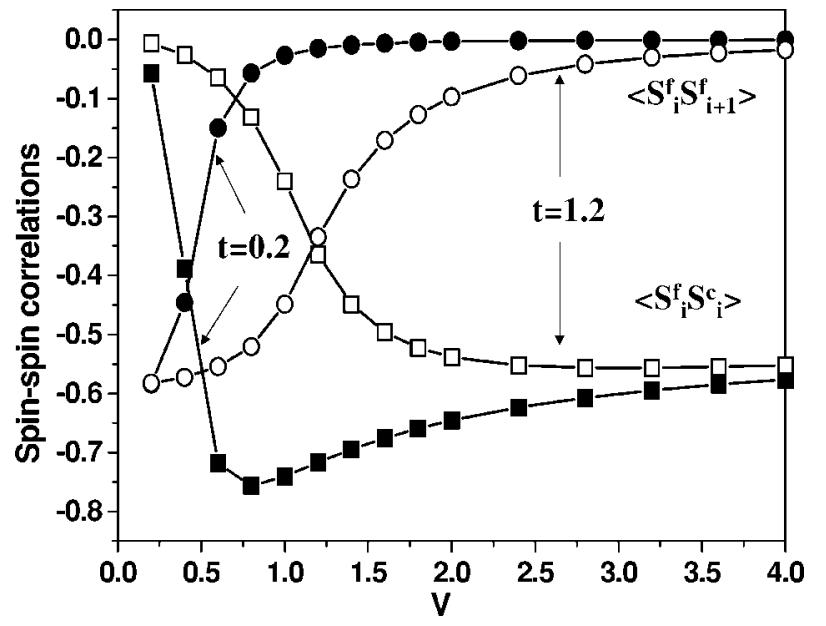

FIG. 1. Nearest-neighbor $f$ - $f$ SCF (circles) and on-site $f$-c SCF (squares) as a function of $V$ for $t=0.2$ (closed symbols) and $t=1.2$ (open symbols), respectively.

ranged in a ring. As expected, the cluster has a singlet ground state $\left(S_{g}=0\right.$, where $S_{g}$ is the ground-state spin) at half filling. The energy spacing in the conduction band is $\Delta=4 t /(N-1)$ $=4 t / 5$. The exact diagonalization calculations employ periodic boundary conditions. At present there is a lack of knowledge how the two energy scales, $T_{K}$ and $T_{R K K Y}$, become modified on going from a bulk system to a cluster with discrete spacing $\Delta$. Thus, we choose to compare the on-site local Kondo spin correlation function (SCF) $\left\langle S_{i, z}^{f} S_{i, z}^{c}\right\rangle$ and the nonlocal (nearest-neighbor) RKKY SCF $\left\langle S_{i, z}^{f} S_{(i+1), z}^{f}\right\rangle$ to assign a state to the Kondo or RKKY regimes, in analogy to mean field treatments. ${ }^{19}$

In Fig. 1 we present the variation of the local Kondo SCF $\left\langle S_{i, z}^{f} S_{i, z}^{c}\right\rangle$ (squares) and the nearest-neighbor RKKY SCF $\left\langle S_{i, z}^{f} S_{(i+1), z}^{f}\right\rangle$ (circles) as a function of hybridization for two values of the hopping matrix element $t=0.2$ (closed symbols) and $t=1.2$ (open symbols), respectively. For weak hybridization the nonlocal (local) nearest-neighbor RKKY (Kondo) SCF is large (small), indicating strong short-range antiferromagnetic coupling between the $f-f$ local moments, which leads to long-range magnetic ordering for extended systems. As $V$ increases, $\left\langle S_{i, z}^{f} S_{(i+1), z}^{f}\right\rangle$ decreases whereas the $\left\langle S_{i, z}^{f} S_{i, z}^{c}\right\rangle$ increases (in absolute value) saturating at large values of $V$. This gives rise to the condensation of independent local Kondo singlets at low temperatures, i.e., a disordered spinliquid phase. For large $V$ the physics are local. Interestingly, as $t$ or $\Delta$ decreases the $f$ - $c$ SCF is dramatically enhanced while the $f-f$ SCF becomes weaker, indicating a transition from the RKKY to the Kondo regime. The nearest-neighbor $f-f$ SCF becomes stronger for small cluster size (large $t$ ) compared to the bulk system because there is no destructive interference of the RKKY coupling at different wave vectors.

In Fig. 2 we present the energy spacing versus $V$ zerotemperature phase diagram of the nanocluster, which illustrates the interplay between Kondo and RKKY interactions. In the RKKY region $\left\langle S_{i, z}^{f} S_{(i+1), z}^{f}\right\rangle$ is larger than $\left\langle S_{i, z}^{f} S_{i, z}^{c}\right\rangle$ and the total local moment squared $\mu^{2}=\left\langle\left(\mu_{f}+\mu_{c}\right)^{2}\right\rangle \neq 0$; in the Kondo regime $\left\langle S_{i, z}^{f} S_{(i+1), z}^{f}\right\rangle$ is smaller than the $\left\langle S_{i, z}^{f} S_{i, z}^{c}\right\rangle, \mu^{2}$

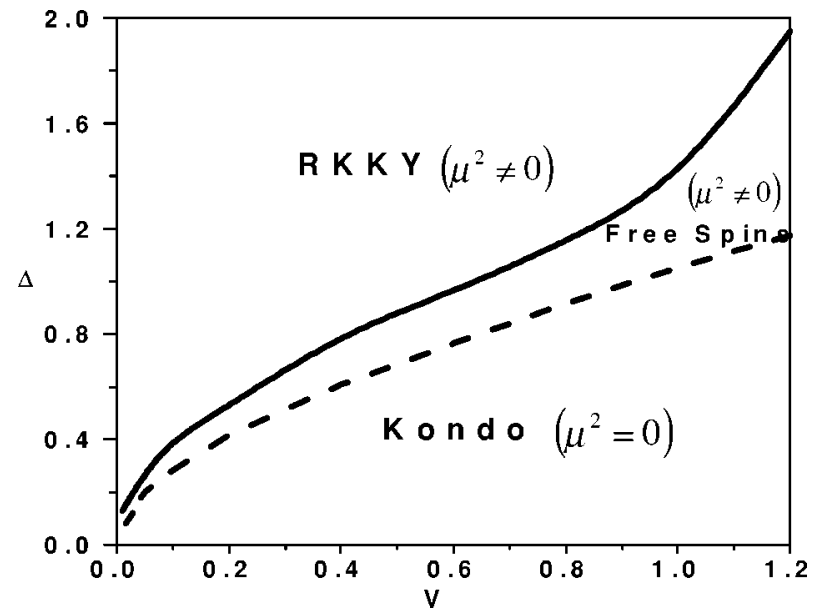

FIG. 2. Energy spacing $\Delta$ vs hybridization zero-temperature phase diagram. The solid curve denotes the crossover point of the spin-spin correlation function in Fig. 1; the dashed curve denotes the set of points where the on-site total moment squared $\left\langle\left(\mu_{f}\right.\right.$ $\left.\left.+\mu_{c}\right)^{2}\right\rangle=0.0 \pm 0.05$.

$=0$ and the ground state is composed of independent local singlets. The solid curve indicates the critical value of the hybridization $V=V_{c}$ or the critical value of energy spacing $\Delta=\Delta_{c}$, where the local and nonlocal SCF's are equal, i.e., $\left\langle S_{i, z}^{f} S_{(i+1), z}^{f}\right\rangle=\left\langle S_{i, z}^{f} S_{i, z}^{c}\right\rangle$. The dashed curve denotes the points where the total local moment square vanishes. Thus, in the intermediate regime, the so-called free spin regime, ${ }^{20}$ $\left\langle S_{i, z}^{f} S_{(i+1), z}^{f}\right\rangle$ is smaller than the $\left\langle S_{i, z}^{f} S_{i, z}^{c}\right\rangle$, the $f$ moment is partially quenched, and $\mu^{2} \neq 0$. Interestingly, we find that the free spin regime becomes narrower as the average level spacing $\Delta$ is reduced. This result may be interpreted as a quantum critical regime for the nanocluster due to the finite energy spacing, which eventually reduces to a quantum critical point when $\Delta \rightarrow 0$.

The $T=0$ exact diagonalization results on small clusters are generally plagued by strong finite size effects. ${ }^{18,21}$ Performing calculations at finite $T$ gives not only the thermodynamic properties of the system, but most importantly diminishes finite-size effects for $k_{B} T \gg \Delta$. In Fig. 3 we show the nearest-neighbor $f$ - $f$ SCF and on-site Kondo $f$ - $c$ SCF as a function of temperature for $t=0.2$ and $V=0.2<V_{c}$ and $V$ $=0.4>V_{c}$, where $V_{c}=0.25$. At high temperatures, the free moments of the $f$ and conduction electrons are essentially decoupled. The nearest-neighbor nonlocal SCF falls more rapidly with $T$ than the on-site local $f$ - $c$ SCF, indicating that the nonlocal spin correlations are destroyed easier by thermal fluctuations. For $V<V_{c}$, the nanocluster is dominated by RKKY interactions at temperatures lower than the crossover temperature, $T_{R K K Y}^{c l}$, which denotes the temperature where the nonlocal and local interaction become equal in the nanocluster. In the infinite system this temperature would denote the ordering Néel temperature. On the other hand, for $V$ $>V_{c}$ the RKKY and Kondo spin correlation functions do not intersect at any $T$, and the physics are dominated by the local Kondo interactions.

In Fig. 4 we present the crossover temperature $T_{R K K Y}^{c l}$ for the cluster as a function of hybridization for different values 


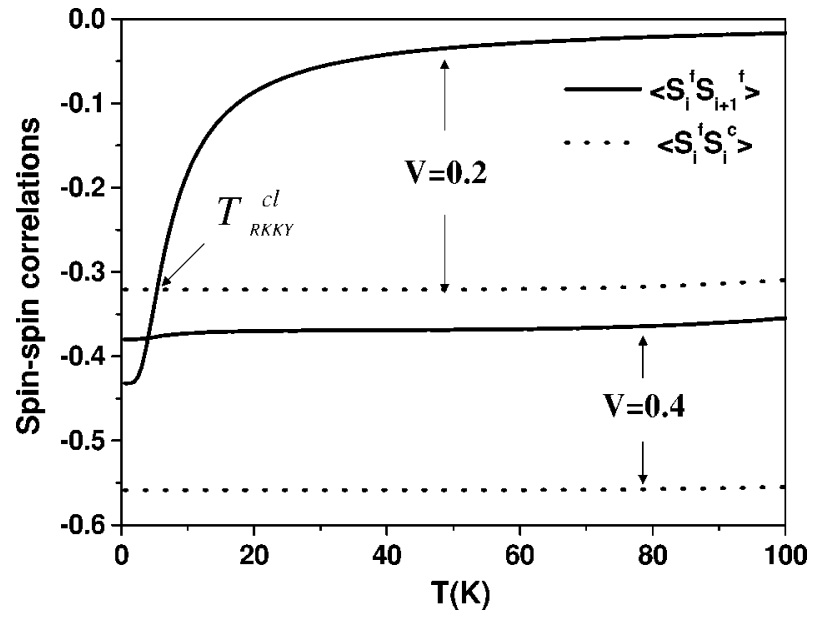

FIG. 3. Nearest-neighbor $f$ - $f$ and on-site $f$ - $c$ SCF vs temperature for $t=0.2$ and $V=0.2<V_{c}$ and $V=0.4>V_{c}$, where $V_{c}=0.25$.

of $t$. This represents the phase diagram of the strongly correlated nanocluster, which is similar to the Doniach phase diagram for the infinite Kondo necklace model. ${ }^{1}$ The phase for $T<T_{R K K Y}^{c l}$ denotes the regime where the nonlocal shortrange magnetic correlations are dominant. For $V<V_{c}$ and $T \gg T_{R K K Y}^{c l}$ one enters into the disordered "free" local moment regime. On the other hand, for $V>V_{c}$ and at low $T$, the nanocluster can be viewed as a condensate of singlets, typical of the Kondo spin-liquid regime. Interestingly, the $T_{R K K Y}^{c l}$ can be tuned by the energy spacing $\Delta$ or the size of the cluster. Thus, increasing $\Delta$ or decreasing the size of the nanocluster results in enhancement of the nonlocal nearestneighbor RKKY correlations and hence $T_{R K K Y}^{c l}$.

In bulk Kondo insulators and heavy-fermion systems, the low- $T$ susceptibility and specific heat behavior is determined by the spin gap, which for the half-filled Anderson lattice model is determined by the ratio of $V$ to $U$. On the other hand, strongly correlated nanoclusters are inherently associ-

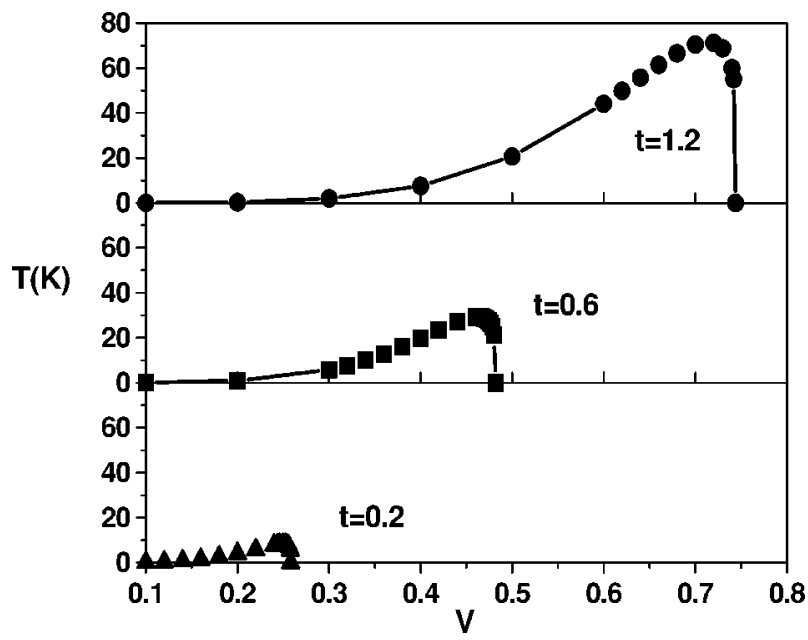

FIG. 4. Effect of energy spacing, $\Delta=4 t /(N-1)$ on the exact Doniach phase diagram for a strongly correlated electron nanochain. The curve represents the crossover temperature $T_{T R K K Y}^{c l}$, where the nonlocal short-range AF spin correlations become equal to the local on-site Kondo spin correlations.

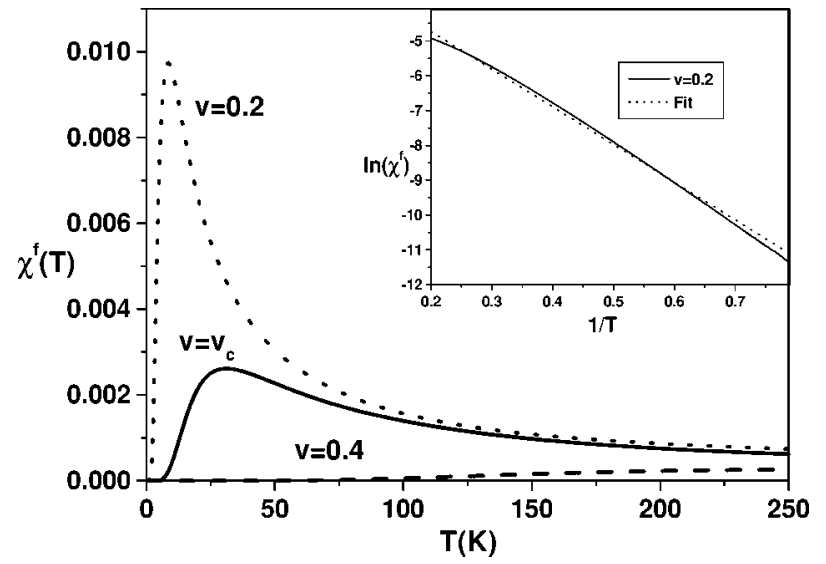

FIG. 5. Local $f$ magnetic susceptibility as a function of temperature for $t=0.2$ and $V=0.2<V_{c}, V=V_{c}=0.25$, and $V=0.4>V_{c}$. The inset shows the linear fit for $\ln \left(\chi^{f}\right)$ vs $1 / T$ at low T.

ated with a new low-energy cutoff, namely, the energy spacing $\Delta$ of the conduction electrons. Thus, a key question is how can the low-temperature physics be tuned by the interplay of the spin gap and the energy spacing? The temperature-dependent local $f$-spin susceptibility, $\chi^{f}(T)$, is

$$
\frac{k_{B} T \chi^{f}(T)}{\left(g \mu_{B}\right)^{2}}=\frac{1}{Q} \sum_{\alpha} e^{-E_{\alpha} / k_{B} T}\left\langle\alpha\left|S^{f}(i) S^{T o t}\right| \alpha\right\rangle,
$$

where $Q$ is the partition function. Here, $S^{T o t}$ is the $z$ projection of the total spin (both the $f$ and $c$ contributions), and $|\alpha\rangle$ and $E_{\alpha}$ are the exact many-body eigenstates and eigenvalues, respectively. When $V=0$, the localized spins and conduction electrons are decoupled and $\chi^{f}(T)$ is simply the sum of the Curie term due to the free $f$ spins and the Pauli term of the free conduction electrons. For finite $V, \chi^{f}(T)$ decreases with temperature at low temperatures. In Fig. 5 we present $\chi^{f}(T)$ as a function of temperature for $t=0.2$ and $V=0.2<V_{c}, V$ $=V_{c}=0.25$, and $V=0.4>V_{c}$. For small $V$ the spin gap, which is smaller than $\Delta$, controls the exponential activation behavior of $\chi^{f}$ at low $T$ (see inset in Fig. 5). In contrast, in the large- $V$ limit, the spin gap becomes larger than $\Delta$ and the low- $T$ behavior of the susceptibility shows no exponential activation. At high $T$ we can see an asymptotic Curie-Weiss regime, typical of localized decoupled moments. The low- $T$ behavior of the susceptibility is associated with the lowestenergy scale determined by the lowest value between the spin gap and the energy spacing $\Delta$. For large values of $t$ (or $\Delta)$ the spin gap is reduced and the spin gap is the lowestenergy scale. Consequently, the low- $T$ behavior exhibits exponential activation associated with the spin gap. Similar low- $T$ behavior is also found for the specific heat. ${ }^{22}$

We have also examined the role of even versus odd numbers of electrons on the magnetic behavior. For $t=1$, changing the number of electrons from $N_{e l}=12$ to $N_{e l}=11$ results in (a) an enhancement of the local Kondo $f$ - $c$ spin correlation function; and (b) a suppression of the nearest-neighbor $f-f$ spin correlation function. This interesting novel tuning of the magnetic behavior can be understood as follows: For an oddelectron cluster, the topmost occupied single-particle energy 
level is singly occupied. On the other hand, for an evenelectron cluster, the topmost occupied single-particle energy level is doubly occupied, thus blocking energy-lowering spin-flip transitions. This energy penalty intrinsically weakens the Kondo correlations. ${ }^{11}$ As expected, changing the number of electrons from even to odd changes $S_{g}=0$ to $S_{g}$ $=\frac{1}{2}$. For $t=0.05$ (Kondo regime), the on site $f-c$ correlation function does not depend as strongly on the parity in the number of electrons because the sites are locked into singlets. On the other hand, the nonlocal RKKY becomes suppressed as in the case of large energy spacing.

Exact diagonalization calculations reveal that the interplay among the energy spacing, the parity of the number of electrons, and the hybridization gives rise to a novel tuning of the magnetic behavior of a dense strongly correlated nanocluster. This interesting and important tuning can drive the nanocluster from the Kondo to the RKKY regime, i.e., a tunable Doniach phase diagram in nanoclusters. For weak hybridization, where the spin gap is smaller than $\Delta$, both the low-temperature local $f$ susceptibility and specific heat exhibit an exponential activation behavior associated with the spin gap. In contrast, in the large hybridization limit, $\Delta$ is smaller than the spin gap, the physics become local, and the exponential activation behavior disappears. We believe that the conclusions of our calculations may be relevant to experimental realizations ${ }^{8}$ of small clusters, to a gas of ultracold atoms, ${ }^{9}$ and quantum computation. ${ }^{10}$

The research at California State University Northridge was supported through NSF under Grant No. DMR-0097187, NASA under Grant No. NCC5-513, and the Keck and Parsons Foundations grants. The calculations were performed on the the CSUN Massively Parallel Computer Platform supported through NSF under Grant No. DMR-0011656.

\footnotetext{
*Email address: yan.luo@csun.edu

${ }^{1}$ S. Doniach, Physica B \& C 91, 231 (1977).

${ }^{2}$ C. M. Varma, Rev. Mod. Phys. 48, 219 (1976).

${ }^{3}$ J. L. Smith and Q. Si, Phys. Rev. B 61, 5184 (2000).

${ }^{4}$ A. C. Hewson, The Kondo Problem to Heavy Fermions (Cambridge University Press, Cambridge, England, 1993).

${ }^{5}$ D. G. Gordon, H. Shtrikman, D. Mahalu, D. A. Magder, U. Meirav, and M. A. Kaster, Nature (London) 391, 156 (1998).

${ }^{6}$ V. V. Madhavan, W. Chen, T. Jamneala, M. F. Crommie, and N. S. Wingreen, Science 280, 567 (1998); T. Jamneala, V. Madhavan, W. Chen, and M. F. Crommie, Phys. Rev. B 61, 9990 (2000).

${ }^{7}$ H. C. Manoharan, C. P. Lutz, and D. M. Eigler, Nature (London) 403, 512 (2000).

${ }^{8}$ T. Odom, J. L. Huang, C. Li Cheung, and C. M. Lieber, Science 290, 1549 (2000), and references therein.

${ }^{9}$ M. Greiner, O. Mandel, T. Esslinger, T. W. Hänsch, and I. Bloch, Nature (London) 415, 39 (2002).

${ }^{10}$ Q. A. Turchette, C. S. Wood, B. E. King, C. J. Myatt, D. Leibfried, W. M. Itano, C. Monroe, and D. J. Wineland, Phys. Rev. Lett. 81, 3631 (1998).

${ }^{11}$ W. B. Thimm, J. Kroha, and J. V. Delft, Phys. Rev. Lett. 82, 2143
}

(1999).

${ }^{12}$ H. Hu, G. M. Zhang, and L. Yu, Phys. Rev. Lett. 86, 5558 (2001).

${ }^{13}$ P. S. Cornaglia and C. A. Balseiro, Phys. Rev. B 66, 115303 (2002).

${ }^{14}$ P. Simon and I. Affleck, Phys. Rev. Lett. 89, 206602 (2002).

${ }^{15}$ P. Schlottmann, Phys. Rev. B 65, 174407 (2002).

${ }^{16}$ G. A. Fiete, G. Zarand, B. I. Halperin, and Y. Oreg, Phys. Rev. B 66, 024431 (2002).

${ }^{17}$ P. Schlottmann, Phys. Rev. B 65, 024431 (2001).

${ }^{18}$ G. M. Pastor, R. Hirsch, and B. Mühlschlegel, Phys. Rev. Lett. 72, 3879 (1994).

${ }^{19}$ B. Coqblin, C. Lacroix, M. S. Gusmao, and J. R. Iglesias, Phys. Rev. B 67, 064417 (2003).

${ }^{20}$ A. Schröder, G. Aeppli, R. Coldea, M. Adams, O. Stockert, H. V. Löhneysen, E. Bucher, R. Ramazashvili, and P. Coleman, Nature (London) 407, 351 (2000).

${ }^{21}$ K. Haule, J. Bonca, and P. Prelovsek, Phys. Rev. B 61, 2482 (2000).

${ }^{22}$ N. Kioussis, Y. Luo, and C. Verdozzi, in Physics of Spin in Solids: Materials, Methods, and Applicaitons, edited by S. Halilov, NATO Science Series, Vol. 156, 115 (2004). 